\title{
Ablation of Copper by a Single Ultrashort Laser Pulse
}

\author{
S.Y. Wang', Y.P. Ren ${ }^{2}$, K.P. Chang ${ }^{1}$, C.W. Cheng ${ }^{3, *}$, J.K. Chen ${ }^{2}$, and D.Y. Tzou ${ }^{2}$ \\ ${ }^{1}$ ITRI Southern Campus, Industrial Technology Research Institute, No. 8, Gongyan Rd., Liujia District, Tainan City 734, \\ Taiwan \\ ${ }^{2}$ Department of Mechanical and Aerospace Engineering, University of Missouri, Columbia, Missouri 65211, U.S.A. \\ ${ }^{3}$ Department of Mechanical Engineering, National Chiao Tung University, No. 1001, Ta Hsueh Road, Hsinchu 30010, Tai- \\ wan \\ E-mail: weicheng@nctu.edu.tw(C.W. Cheng)
}

\begin{abstract}
Thermal ablation of copper films by a single Ti:Sapphire femtosecond laser pulse of wavelength $800 \mathrm{~nm}$ and duration $100 \mathrm{fs}$ was investigated experimentally and theoretically. The laser experiments were performed; the ablation depth and crater profiles were measured for laser fluences up to $1037 \mathrm{~J} / \mathrm{cm}^{2}$. A comprehensive axisymmetric model, including a two-temperature model, phase change models for rapid melting and evaporation, and a phase explosion model for ejecting metastable liquid and vapor, was developed to simulate the laser material ablation process. The simulated ablation depths and crater profiles agree well with the experimental measurements.
\end{abstract}

DOI: $10.2961 /$ ilmn.2014.02.0002

Keywords: femtosecond laser, axisymmetric model, thermal ablation, copper film

\section{Introduction}

Femtosecond laser material processing has been demonstrated as an effective means for micro/nano machining and surface structural modification of solid materials because of its minimal heat affected zone, less debris contamination, and good reproducibility [1-3]. A great number of experimental and theoretical works on ultrashort lasermaterial interactions have been reported since 1990s. Most of them are focused on thermal transport using a twotemperature model $[4,5]$ or time-resolved measurements $[6,7]$. In the study of ultrafast laser material ablation, many experimental works are dedicated to machining grooves. The ablation depth per pulse (unit: $\mu \mathrm{m} /$ pulse) was thus averaged by dividing the total groove depth by the equivalent number of pulses based on a single irradiation spot assumption [1, 8-11]. In addition, the ablation rate per pulse (unit: $\mu \mathrm{m}^{3} /$ pulse) was further averaged by dividing the total ablated material volume by the equivalent number of pulses [12]. However, some studies [13-15] have shown that the ablation fluence threshold by a single laser pulse is generally larger than the values averaged from multiple laser pulses. Due to the numerical challenge in both space and time scales, the theoretical s DOI: 10.2961/jlmn.2014.02.0001 using a 1D laser ablation modes aiong witn tne singie snor assumption [9-11, 16-18]. Several questions thus arise on whether the 1D approach is well suited for multi-pulse laser ablation, including the optical model that refers to a fresh undamaged surface, laser beam reflection due to inclined groove walls, plasma shielding, incubation effect, etc. When the laser spot size is smaller or comparable to the heat-affected zone size of interest, use of the simplified 1D approach may not be sufficient. Instead, a higher dimensional model should be considered so that the thermal and ablation response can be accurately described.
In this paper, material ablation experiments of copper films are conducted for a single femtosecond laser pulse of different powers. A comprehensive axisymmetric model, including a two-temperature model, two phase change models for rapid melting and vaporization, and a phase explosion model, is developed. The experimental and simulation results of ablation depth and crater shapes are compared. The $1 \mathrm{D}$ result is also presented for comparison.

\section{Experimental}

A commercial copper film (GTJ-MP, FCFT) of thickness $35 \mu \mathrm{m}$ was irradiated in air by a single pulse of $100 \mathrm{fs}$, 800-nm Ti:Sapphire laser. The laser beam was linearly polarized and spatially filtered, resulting in an essentially Gaussian profile. The energy of the laser beam was attenuated by a rotatable half-waveplate and a polarizing beam splitter. The transmitted component of the laser beam was incident onto a beam splitter, the reflected beam was directed to a power detector, and the laser irradiation energy on the copper target was measured. Meanwhile, the transmitted linearly-polarized laser beam was passed through a shutter and a series of reflective mirrors, and subsequently

sbjective lens (numerical aperture 0.26, M Plan Apo NIK, Iviitutoyo). The position of the objective lens was adjusted in the vertical direction (i.e. $z$-axis). The material process was continuously monitored via a co-axial CCD camera system. The laser powers applied were in the range of $0.005 \sim 0.17 \mathrm{~W}$ at a repetition rate of $1 \mathrm{kHz}$. To make a single shot measurement, the sample was translated using an X-Y motion stage, and the copper film was then drilled in an array pattern $(7 \times 7)$ under the same laser fluence. The morphology of the generated craters on the copper film was captured using a scanning electron microscopy (SEM, Hitachi S-4700) from the top-view. The sample was then sec- 
tioned and placed on the silicon wafer substrate and embedded in the epoxy. The sample was further mechanical polished through the center of a crater cross-sectionally. The crater shapes, including the diameters, the maximum depths, and crater profiles were measured from the crosssection SEM image.

\section{Modeling and Simulation}

Consider that a copper film at initial temperature $T_{i}$ is normally irradiated by a single Gaussian laser pulse on the front surface $(z=0)$. The axisymmetric two-temperature model is given by:

$$
\begin{gathered}
C_{e} \frac{\partial T_{e}}{\partial t}=\left[\frac{1}{r} \frac{\partial}{\partial r}\left(k_{e} r \frac{\partial T_{e}}{\partial r}\right)+\frac{\partial}{\partial z}\left(k_{e} \frac{\partial T_{e}}{\partial z}\right)\right] \\
-G\left(T_{e}-T_{l}\right)+S \\
C_{l} \frac{\partial T_{l}}{\partial t}=\left[\frac{1}{r} \frac{\partial}{\partial r}\left(k_{l} r \frac{\partial T_{l}}{\partial r}\right)+\frac{\partial}{\partial z}\left(k_{l} \frac{\partial T_{l}}{\partial z}\right)\right]+G\left(T_{e}-T_{l}\right)
\end{gathered}
$$

where $T$ is temperature, $t$ is time, $C$ is heat capacity, $k$ is thermal conductivity, $G$ is electron-phonon coupling factor, and $r$ is coordinate in the radial direction. The subscripts $e$ and $l$ denote electron and lattice, respectively. The laser heat density $S$ in Eq. (1) can be expressed as:

$$
\begin{aligned}
& S(r, z, t)= \sqrt{\frac{\beta}{\pi}} \frac{[1-R(r, 0, t)] J_{o}}{t_{p}} \frac{1}{\delta(r, z, t)+\delta_{b}(r, z, t)} \\
& \exp \left[-2\left(\frac{r}{r_{o}}\right)^{2}-\int_{0}^{z} \frac{1}{\delta+\delta_{b}} d z-\beta\left(\frac{t}{t_{p}}\right)^{2}\right]
\end{aligned}
$$

where $R(r, 0, t)$ is surface reflectivity, $t_{p}$ laser pulse full width at half maximum (FWHM), $J_{o}$ laser fluence, $\alpha=1 / \delta$ absorption coefficient, $\delta_{b}$ electron ballistic range, $r_{o}$ the characteristic spot radius of laser pulse defined at $e^{-2}$, and $\beta$ $=4 \ln (2)$. The central line of laser beam coincides with the line $r=0$. The lasing starts from $t=-2 t_{p}$, reaches its peak power at $t=0$, and ends at $t=2 t_{p}$. The laser energy outside this time period is neglected because it is too small to significantly alter the results. The electron ballistic range $\delta_{b}$ is added to take into account the effects of the ballistic motion of photon-excited electrons that spreads the absorbed laser energy into a deeper part of material. A constant value of $\delta_{b}$ is often computed based on the assumption that the excited, nonequilibrium electrons could penetrate into the nonexcited region at the Fermi velocity for 100 fs [19], or is arbitrarily assumed [13]. In this work, it is determined by $\delta_{b}=v_{e} \times t_{b}$, where $t_{b}=80 \mathrm{fs}$, and $v_{e}$ is the electron velocity calculated by

$$
v_{e}=\sqrt{3 k_{B} T_{e} / m_{e}}
$$

where $k_{B}$ is Boltzmann constant, and $m_{e}$ is mass of an electron.

Optical properties are of paramount importance for laser energy deposition and the resulting thermal response. Recently, Ren et al. [20] proposed an extended Drude model which can accurately characterize the reflectivity and absorption coefficient for gold in the range of wavelengths $500-1000 \mathrm{~nm}$. That model is modified here to account for the temperature-dependent interband transition effect as follows:

$$
\varepsilon(\omega)=\varepsilon_{\infty}-\frac{\omega_{p}^{2}}{\omega\left(\omega+i \gamma_{D}\right)}-\frac{f \Omega_{L}^{2}}{\left(\omega^{2}-\Omega_{L}^{2}\right)+i A \gamma_{D} \omega}=\varepsilon_{1}+i \varepsilon_{2}
$$

where $\varepsilon_{\infty}$ is dielectric constant, $\omega_{p}$ plasma frequency, $\omega$ laser frequency, $\gamma_{D}$ damping coefficient which equals reciprocal of electron relaxation time $\left(\tau_{e}\right), f$ a weighting factor, $\Omega_{L}$ oscillator strength of the Lorentz oscillators, and $A$ a constant. The following values are optimized for copper with the reflectivity and absorption coefficient at room temperature for wavelengths 600-1000 nm: $\varepsilon_{\infty}=9.429, \omega_{p}$ $=1.359 \times 10^{16} \mathrm{~Hz}, \Omega_{L}=1.767 \times 10^{15} \mathrm{~Hz}, f=3.636$, and $A=$ 44.53 .

The optical properties $R$ and $\alpha$ can be determined by Fresnel function [21]:

$$
R(r, z, t)=\frac{\left(f_{1}-1\right)^{2}+f_{2}^{2}}{\left(f_{1}+1\right)^{2}+f_{2}^{2}} ; \alpha(r, z, t)=\frac{2 \omega f_{2}}{c}
$$

where $c$ is light speed in vacuum, and the normal refractive index $\left(f_{1}\right)$ and extinction coefficient $\left(f_{2}\right)$ are:

$$
f_{1}(r, z, t)=\sqrt{\frac{\varepsilon_{1}+\sqrt{\varepsilon_{1}^{2}+\varepsilon_{2}^{2}}}{2}} ; \quad f_{2}(r, z, t)=\sqrt{\frac{-\varepsilon_{1}+\sqrt{\varepsilon_{1}^{2}+\varepsilon_{2}^{2}}}{2}}
$$

The electron relaxation time, which is the reciprocal of the damping coefficient $\gamma_{D}$ in Eq. (5), is given by:

$$
\tau_{e}=\frac{1}{1.414 v_{e, p}+A_{e} T_{e}^{2}}
$$

with the electron-phonon collision rate $v_{e, p}$ depending on both electron and lattice temperature [22]:

$$
\begin{aligned}
\mathrm{v}_{e, p} & =\frac{\Xi^{2}}{8 \pi \varepsilon_{F} v_{F} \rho s} \frac{m_{\text {opt }}}{m_{e}}\left\{\int_{0}^{q_{b}} \frac{e^{\phi_{l}}+e^{\phi_{e}}}{\left(e^{\phi_{l}}-1\right)\left(e^{\phi_{e}}+1\right)} q^{4} d q\right. \\
& +\eta \int_{0}^{q_{b}} \frac{e^{\phi_{l}}-e^{\phi_{e}}}{\left(e^{\phi_{l}}-1\right)\left(e^{\phi_{e}}+1\right)} q^{3} d q+q_{b} \frac{e^{\varphi_{l}}+e^{\varphi_{e}}}{\left(e^{\varphi_{l}}-1\right)\left(e^{\varphi_{e}}+1\right)} \\
& \left.\times \frac{\left(2 v_{F}\right)^{4}-q_{b}^{4}}{4}-4 \eta q_{b}^{2} v_{F}^{2} \frac{e^{\varphi_{l}}-e^{\varphi_{e}}}{\left(e^{\varphi_{l}}-1\right)\left(e^{\varphi_{e}}+1\right)}\right\}
\end{aligned}
$$

where $A_{e}$ is the material constants for electron relaxtion time, $\varepsilon_{F}$ is Fermi energy, $v_{F}$ Fermi velocity, $\rho$ mass density, $s$ longitudinal sound velocity, $m_{\text {opt }}$ effective electron mass, $m_{e}$ mass of an electron, $q$ phonon wave vector, $q_{b}$ maximum phonon wave vector, $\phi_{e}=\beta_{e} \hbar q s$ with Planck's constant $h=2 \pi \hbar$ and $\beta_{e}=T_{e} / k_{B}$ with $k_{B}$ being Boltzmann's constant, $\phi_{l}=\beta_{l} \hbar q s$ with $\beta_{l}=T_{l} / k_{B}, \varphi_{e}=\beta_{e} \hbar q_{b} s, \varphi_{l}=\beta_{l} \hbar q_{b} s$, and $\eta=2 m_{\text {opt }} s / \hbar$. For copper, $A_{e}$ in Eq. (8) and $\Xi, q_{b}$ and $m_{\text {opt }}$ in Eq. (9) are: $1.75 \times 10^{7} \mathrm{~K}^{-2} \mathrm{~s}^{-1}, 3.99 \mathrm{eV}, 8.97 \times 10^{9} \mathrm{~m}^{-1}$, and $1.39 m_{e}$, respectively. The constant value of 1.414 in Eq. (8) is set for which the electron relaxation time of copper at room temperature $\left(T_{l}=T_{e}=300 \mathrm{~K}\right)$ is $25 \mathrm{fs}$ [21].

If the fluence of a femtosecond laser pulse is sufficiently high, a solid medium can be melted and ablated through vaporization and even by phase explosion. To accurately simulate this ultrafast thermal ablation process, the above axisymmetric two-step heating model is integrated with two phase change models [23] for ultrafast melting/resolidification and evaporation and a phase explosion model for ejection of metastable liquid and vapor $[11,17$, 24].

In a superheating and undercooling process, the solidliquid interfacial velocity is controlled by nucleation dy- 
namics and the temperature can be computed with the energy balance equation. In the ultrafast vaporization, the liquid/vapor interfacial velocity is regulated by the nonequilibrium, superheating kinetics of volume vaporization and the temperature can be determined by the energy balance equation. The velocity of dynamics vaporization is limited by the characteristic speed of sound at the liquidus line [23]. Because the process time is too short for the necessary heterogeneous nuclei to form, the melted material can be heated well beyond the normal boiling point without boiling. As the temperature of the superheated liquid approaches the thermodynamic equilibrium critical temperature $\left(T_{t c}\right)$, the tensile strength of the liquid rapidly falls to zero. Consequently, homogeneous bubble nucleation occurs at an extremely high rate. The superheated liquid thus relaxes explosively into a mixture of vapor and equilibrium liquid droplets and immediately ejected from the bulk material. The details of these phase change models aforementioned and the nonlinear numerical algorithms for simulating the melting/resolidification and vaporization can be found in Ren et al. [23] and the references cited in that paper. They are not described here for brevity.

In the numerical analysis, a copper film of $15 \mu \mathrm{m}$ in thickness and $15 \mu \mathrm{m}$ in radius is modeled. A finite difference with non-uniform mesh is adopted. The smallest grid sizes are $1 \mathrm{~nm}$ in the thickness direction and $2 \mathrm{~nm}$ in the radial direction. The difference of any two consecutive grid sizes is $0.25 \mathrm{~nm}$, stemming from the central point $(0,0)$ in both the thickness and radial directions. The number of the total grid points meshed is 83,592 . The thermophysical properties employed can be found in Reference [25]. When the calculated liquid lattice temperature at a grid point reaches to $90 \%$ of the thermodynamic equilibrium critical temperature $\left(T_{t c}=7696 \mathrm{~K}\right.$ for copper [26]), the superheated liquid is assumed to undergo phase explosion. That grid point is then removed from the model. The credibility of the computer code is first validated with the $1 \mathrm{D}$ ablation results [11] by setting $r_{o}$ to be $10 \mathrm{~cm}$ with which the film sample can be considered to be uniformly heated by a flattop laser pulse.

\section{Results and Discussion}

Figure 1 presents the top view and the cross-section SEM images of the craters on the copper sample generated by four laser pulses of different fluences from $61 \mathrm{~J} / \mathrm{cm}^{2}$ to $427 \mathrm{~J} / \mathrm{cm}^{2}$. The re-casted splash around the craters may result mainly from the phase explosion. As expected, both the diameter and depth of the craters increase with increasing laser power. The laser focus spot size at the sample surface was determined by plotting the square of the craters diameter against laser energy in logarithmic scale [27]. The laser beam focus diameter obtained was $3.25 \mu \mathrm{m}$ at $e^{-2}$ which is close to the theoretical focus spot size at $3.75 \mu \mathrm{m}$ (1.22 $\lambda / \mathrm{NA}, \lambda=800 \mathrm{~nm}$ is the wavelength, $\mathrm{NA}=0.26$ is the numerical aperture).

Comparison of the maximum ablation depths between the experimental measurements and the simulations (at the center of the craters) is given in Fig. 2. It can be observed that the present experimental ablation depths resulting from the single shot at very high fluences are about 2 4 times the averaged depths obtained from line scanning grooves machined by multiple pulses $[8,9,11]$. Apparently, the axisymmetric model simulations are in fairly good correlation with the experimental data for laser fluences up to $1037 \mathrm{~J} / \mathrm{cm}^{2}$ studied in this work. Although we did not perform the ablation tests for the low fluences, the simulated ablation depth may also agree well for the range of low fluence, indicated by the ablation threshold of $1.06 \mathrm{~J} / \mathrm{cm}^{2}$ which was extrapolated from the experimental data of ablation diameters in the range of relatively low fluences $1.2 \sim$ $20 \mathrm{~J} / \mathrm{cm}^{2}$ [15]. For those very high laser fluences, the material ablation through vaporization is very small compared to that through phase explosion. The ablation depths simulated with the 1D model are quite close to those simulated with the axisymmetric model, except for the very high fluences where the differences are approximately $10 \%$. It is suggested that the laser ablation tests be conducted for better validation of the present numerical model.
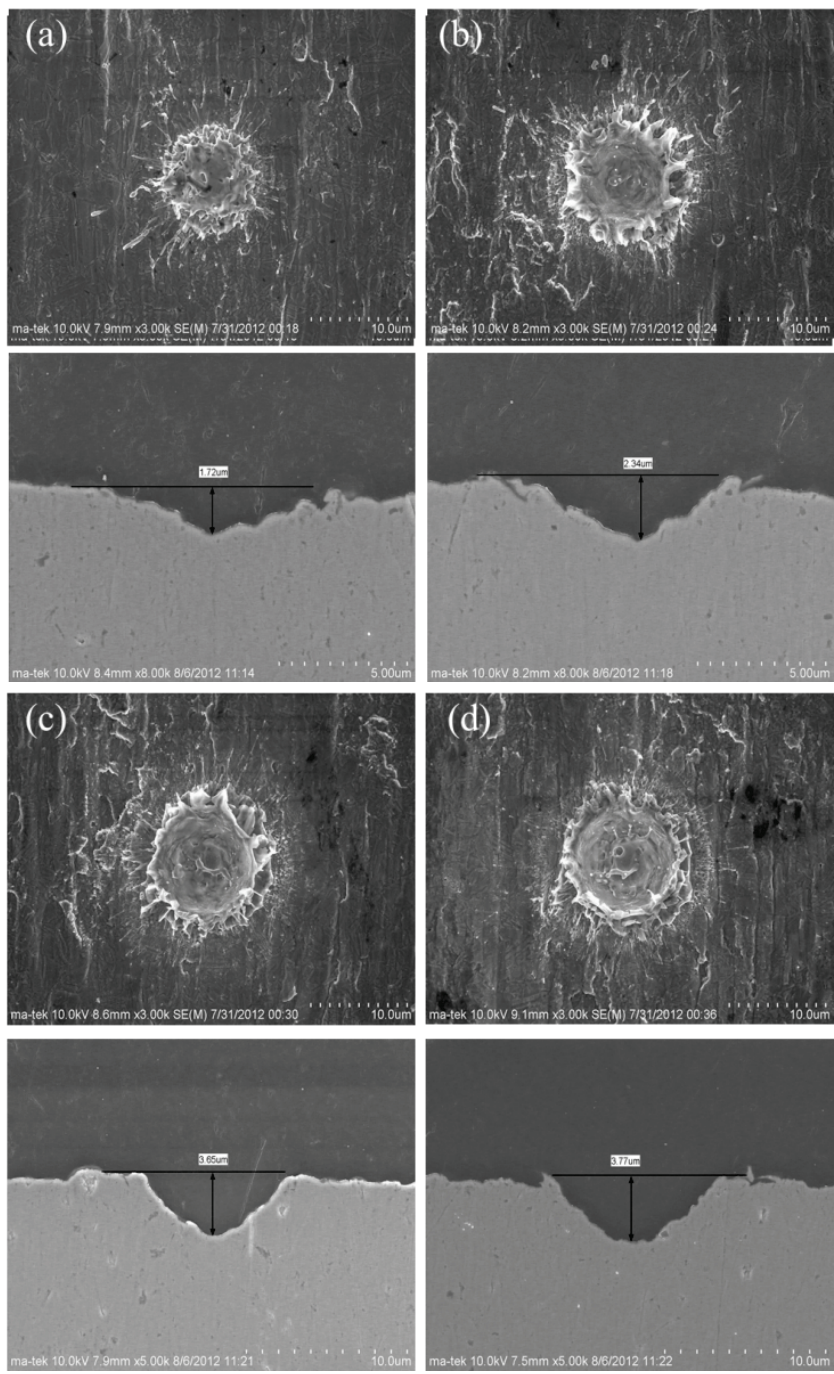

Fig. 1 Craters and their cross-section profiles created by the laser pulses at fluence: (a) $61 \mathrm{~J} / \mathrm{cm}^{2}$, (b) $183 \mathrm{~J} / \mathrm{cm}^{2}$, (c) 305 $\mathrm{J} / \mathrm{cm}^{2}$, and (d) $427 \mathrm{~J} / \mathrm{cm}^{2}$.

Figure 3 compares the crater shapes for the three different laser fluences, 183, 549 and $1037 \mathrm{~J} / \mathrm{cm}^{2}$. Experimental crater profiles were measured from the crosssection SEM images, e.g. $183 \mathrm{~J} / \mathrm{cm}^{2}$ of Fig. 1(b). At every measurement step of $r=0.5 \mu \mathrm{m}$, the value ablation depth 
was image measured from the base line. The measurement error is believed to be less than $20 \mathrm{~nm}$. It appears that the simulated crater shapes correlate well with the experimental measurements. The asymmetric shapes founded from the experiment could be attributed to non-perfect Gaussian beam profiles due to optical defects or non-linear optical effect.

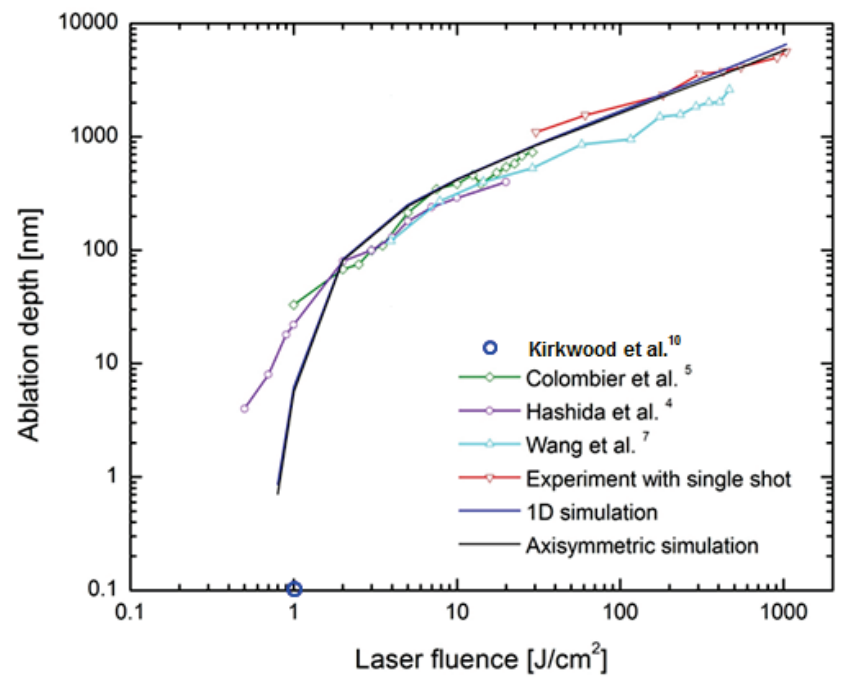

Fig. 2 Comparison of maximum ablation depth between experiment measurements and simulations.

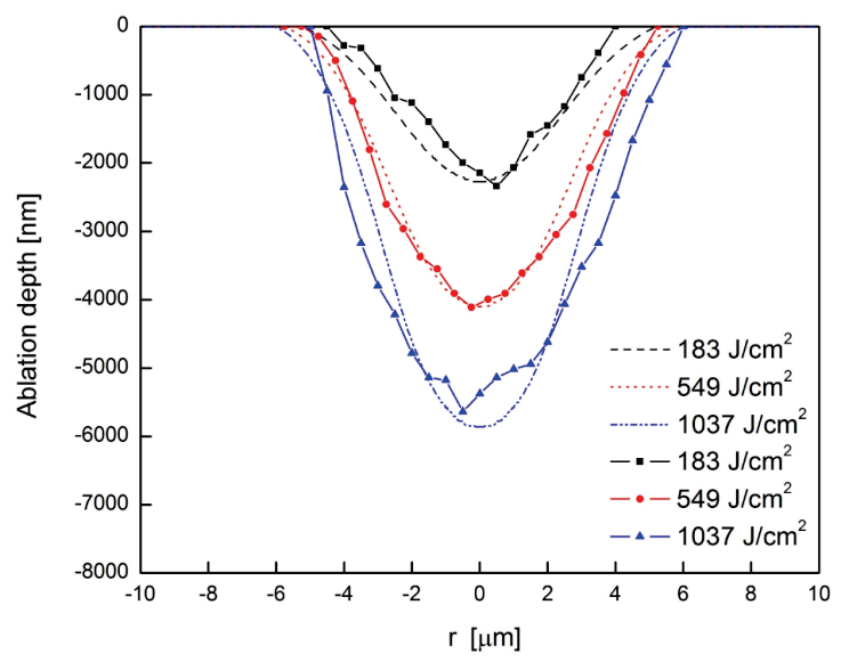

Fig. 3 Comparison of the crater profiles for three different laser fluences: dashed lines - simulation and solid lines with symbols - experiment.

The discrepancy between the axisymmetric and 1D model results can be pronounced if laser spot sizes are smaller than or comparable to the depth of heat affected zone. Figure 4 shows the comparison of ablation depth between the two models for the laser spot size of $0.5 \mu \mathrm{m}$ in diameter and fluence of $549 \mathrm{~J} / \mathrm{cm}^{2}$. For example, the ablation depths obtained from the axisymmetric and 1D model are $215 \mathrm{~nm}$ and $425 \mathrm{~nm}$ for the laser fluence $10 \mathrm{~J} / \mathrm{cm}^{2}$ and $2,237 \mathrm{~nm}$ and 4,491 $\mathrm{nm}$. In this case, the 1D result is approximately double of the axisymmetric result.

The smaller ablation depths simulated with the axisymmetric model can be attributed to the rapid loss of thermal energy in the region underneath the laser spot due to lateral heat conduction. It should be noted that the small spot size of $0.5 \mu \mathrm{m}$ considered here is smaller than that of the diffraction limited, i.e. $3.75 \mu \mathrm{m}(1.22 \lambda / \mathrm{NA}, \lambda=800 \mathrm{~nm}$ is the wavelength, $\mathrm{NA}=0.26$ is the numerical aperture). We believe that a laser spot with size beyond the diffraction limited can be achieved by using near-field optics. Further experimental and theoretical investigations on the nanosize laser spots are suggested.

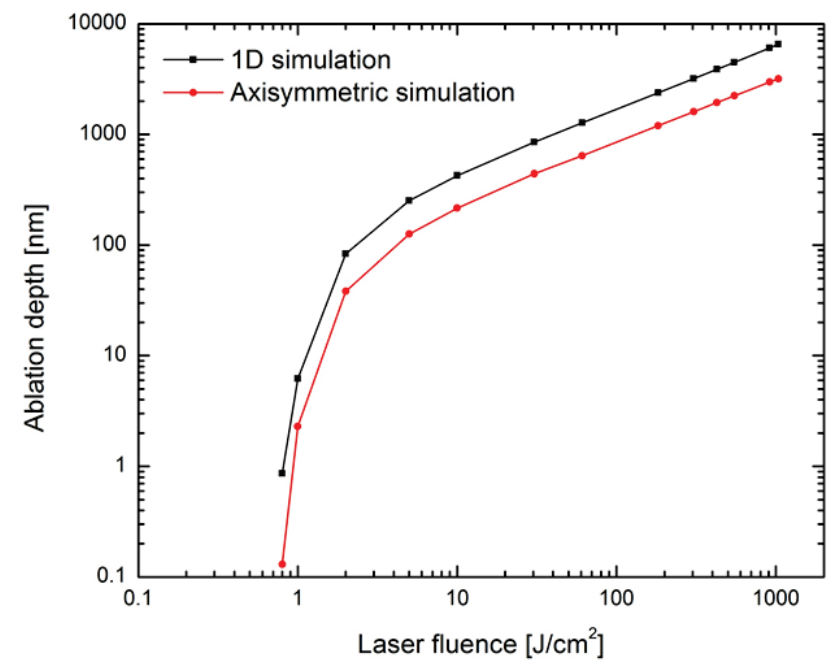

Fig. 4 Comparison of ablation depth simulated with the axisymmetric and 1D model for the laser spot size of 0.5 $\mu \mathrm{m}$ in diameter and fluence of $549 \mathrm{~J} / \mathrm{cm}^{2}$.

\section{Conclusions}

In this paper, new experimental and theoretical results of thermal ablation of copper films were reported for the single femtosecond laser pulse with fluences up to 1037 $\mathrm{J} / \mathrm{cm}^{2}$. It was found from the experiment that the ablation depth resulting from a single laser pulse is much greater than the averaged depth of line scanning grooves machined by multiple pulses. Both the ablation depth and crater diameter increase with laser fluence, as expected. To simulate the laser material ablation process, an axisymmetric comprehensive model, including a two-temperature model for temperature response, two phase change models for rapid melting and evaporation, and a phase explosion model for ejecting metastable liquid and vapor, was developed and parallelized. The simulated ablation depth and crater shapes agree well with the experimental measurements. On the other hand, the accuracy of the ablation depths obtained from the simplified 1D model depends on the laser spot size.

\section{References}

[1] S. Nolte, C. Momma, H. Jacobs et al., "Ablation of metals by ultrashort laser pulses," Journal of the Optical Society of America B-Optical Physics, vol. 14, no. 10, pp. 2716-2722, Oct, 1997.

[2] L. T. Qi, K. Nishii, and Y. Namba, "Regular subwavelength surface structures induced by femtosecond laser pulses on stainless steel," Optics Letters, vol. 34, no. 12, pp. 1846-1848, 2009. 
B. K. Nayak, and M. C. Gupta, "Self-organized micro/nano structures in metal surfaces by ultrafast laser irradiation," Optics and Lasers in Engineering, vol. 48, no. 10, pp. 940-949, 2010.

[4] B. L. K. S. I. Anisimov, and T. L. Perel'man, "Electron emission from metal surfaces exposed to ultrashort laser pulses," Soviet Physics, Journal of Experimental and Theoretical Physics, vol. 39, pp. 375-377, 1974.

[5] T. Q. Qiu, and C. L. Tien, "HEAT-TRANSFER MECHANISMS DURING SHORT-PULSE LASER-HEATING OF METALS," Journal of Heat Transfer-Transactions of the Asme, vol. 115, no. 4, pp. 835-841, Nov, 1993.

[6] J. Konig, S. Nolte, and A. Tunnermann, "Plasma evolution during metal ablation with ultrashort laser pulses," Optics Express, vol. 13, no. 26, pp. 10597-10607, Dec, 2005.

[7] N. Zhang, W. T. Wang, X. N. Zhu et al., "Investigation of ultrashort pulse laser ablation of solid targets by measuring the ablation-generated momentum using a torsion pendulum," Optics Express, vol. 19, no. 9, pp. 8870-8878, Apr, 2011.

[8] M. Hashida, A. F. Semerok, O. Gobert et al., "Ablation threshold dependence on pulse duration for copper," Applied Surface Science, vol. 197, pp. 862-867, Sep 30, 2002.

[9] J. P. Colombier, P. Combis, F. Bonneau et al., "Hydrodynamic simulations of metal ablation by femtosecond laser irradiation," Physical Review B, vol. 71, no. 16, Apr, 2005.

[10] J. Byskov-Nielsen, J.-M. Savolainen, M. S. Christensen et al., "Ultra-short pulse laser ablation of copper, silver and tungsten: experimental data and two-temperature model simulations," Applied Physics a-Materials Science \& Processing, vol. 103, no. 2, pp. 447-453, May, 2011.

[11] S. Y. Wang, Y. Ren, C. W. Cheng et al., "Micromachining of copper by femtosecond laser pulses," Applied Surface Science, vol. 265, pp. 302-308, Jan, 2013.

[12] J. Cheng, W. Perrie, S. P. Edwardson et al., "Effects of laser operating parameters on metals micromachining with ultrafast lasers," Applied Surface Science, vol. 256, no. 5, pp. 1514-1520, Dec, 2009.

[13] J. Byskov-Nielsen, J. M. Savolainen, M. S. Christensen et al., "Ultra-short pulse laser ablation of metals: threshold fluence, incubation coefficient and ablation rates," Applied Physics aMaterials Science \& Processing, vol. 101, no. 1, pp. 97-101, Oct, 2010.

[14] L. Caneve, F. Colao, R. Fantoni et al., "Laser ablation of copper based alloys by single and double pulse laser induced breakdown spectroscopy," Applied Physics a-Materials Science \& Processing, vol. 85, no. 2, pp. 151-157, Nov, 2006.

[15] S. E. Kirkwood, A. C. van Popta, Y. Y. Tsui et al., "Single and multiple shot near-infrared femtosecond laser pulse ablation thresholds of copper," Applied Physics a-Materials Science \& Processing, vol. 81, no. 4, pp. 729-735, Sep, 2005.

[16] J. Yang, Y. Zhao, and X. Zhu, "Theoretical studies of ultrafast ablation of metal targets dominated by phase explosion," Applied Physics a-Materials Science \& Processing, vol. 89, no. 2, pp. 571-578, Nov, 2007.

[17] J. E. B. J. K. Chen, and D. Y. Tzou, "Investigation of ultrafast laser ablation using a semiclassical two-temperature model," Journal of Directed Energy, vol. 1, pp. 261-274, 2005.

[18] J. Cheng, W. Perrie, M. Sharp et al., "Single-pulse drilling study on $\mathrm{Au}, \mathrm{Al}$ and $\mathrm{Ti}$ alloy by using a picosecond laser," Applied Physics a-Materials Science \& Processing, vol. 95, no. 3, pp. 739-746, Jun, 2009

[19] S. S. Wellershoff, J. Hohlfeld, J. Gudde et al., "The role of electron-phonon coupling in femtosecond laser damage of metals," Applied Physics a-Materials Science \& Processing, vol. 69 pp. S99-S107, Dec, 1999.

[20] Y. P. Ren, J. K. Chen, Y. W. Zhang et al., "Ultrashort laser pulse energy deposition in metal films with phase changes," Applied Physics Letters, vol. 98, no. 19, May, 2011.

[21] M. Fox, Optical Properties of Solids: Oxford Univer-sity Press, Oxford, 2010.

[22] D. Fisher, M. Fraenkel, Z. Henis et al., "Interband and intraband (Drude) contributions to femtosecond laser absorption in aluminum," Physical Review E, vol. 65, no. 1, Jan, 2002.

[23] J. K. C. Yunpeng Ren, and Yuwen Zhang, "Modeling of ultrafast phase changes in metal films irradiated by an ultrashort laser pulse using a semiclassical two-temperature model," International Journal Heat and Mass Transfer, vol. 55, pp. 1260-1627, 2012.

[24] R. K. a. A. Miotello, "Comments on explosive mechanisms of laser sputtering," Applied Surface Science, vol. 96-98, pp. 205-215, 1996.

[25] Y. P. Ren, J. K. Chen, and Y. W. Zhang, "Optical properties and thermal response of copper films induced by ultrashort-pulsed lasers," Journal of Applied Physics, vol. 110, no. 11, Dec, 2011.

[26] H. Hess, "Critical data and vapor pressures of aluminium and copper," Zeitschrift für Metallkunde, vol. 89, pp. 388-393, 1998.

[27] J. M. Liu, "SIMPLE TECHNIQUE FOR MEASUREMENTS OF PULSED GAUSSIANBEAM SPOT SIZES," Optics Letters, vol. 7, no. 5, pp. 196-198, 1982.

(Received: July 18, 2013, Accepted: March 10, 2014) 